\title{
The effect of changing topography on the coordinated marching of locust nymphs
}

\author{
Guy Amichay ${ }^{1}$, Gil Ariel ${ }^{2}$, Amir Ayali ${ }^{\text {corresp. } 1,3}$ \\ 1 Department of Zoology, Tel Aviv University, Tel Aviv, Israel \\ 2 Department of Mathematics, Bar-Ilan University, Ramat Gan, Israel \\ 3 Sagol School of Neuroscience, Tel Aviv University, Tel Aviv, Israel \\ Corresponding Author: Amir Ayali \\ Email address: ayali@post.tau.ac.il
}

Collective motion has traditionally been studied in the lab in homogeneous, obstacle-free environments, with little work having been conducted with changing landscapes or topography. Here, the impact of spatial heterogeneity on the collective motion exhibited by marching desert locust nymphs was studied under controlled lab conditions. Our experimental circular arenas, incorporating a funnel-like narrowing followed by rewidening, did not constitute a major barrier to the locusts; but, rather, mimicked a changing topography in the natural environment. We examined its effects on macroscopic features of the locust collective behavior, as well as the any changes in their marching kinematics. A major finding was that of the limited extent to which the changing topography affected system-level features of the marching locust group, such as the order parameter and the fraction of walking individuals, despite increased crowding at the funnel. Overall, marching kinematics was also very little affected, suggesting that locust marching bands adjust to the environment, with little effect on the overall dynamics of the group. These findings are in contrast to recent theoretical results predicting that environmental heterogeneities qualitatively alter the dynamics of collectively moving particles; and highlight the crucial role of rapid individual plasticity and adaptability in the dynamics of flocks and swarms. Our study has revealed other important features of the marching behavior of the desert locust in addition to its robustness: the locusts demonstrated both, clear thigmotaxis and a tendency to spread-out and fill the available space. 
1 The effect of changing topography on the coordinated marching of locust nymphs

2

$5 \quad{ }^{1}$ Department of Zoology, Faculty of Life Sciences, Tel Aviv University, 6997801, Israel.

$6 \quad{ }^{2}$ Department of Mathematics, Bar Ilan University, Ramat-Gan 52900, Israel.

$7 \quad{ }^{3}$ Sagol School of Neuroscience, Tel Aviv University, 6997801, Israel.

8

9

10

11

12

*Corresponding authors: ayali@post.tau.ac.il; arielg@math.biu.ac.il

$18 \uparrow$ Current address: The Department of Collective Behaviour at the University of Konstanz/Max 
21 Abstract

22 Collective motion has traditionally been studied in the lab in homogeneous, obstacle-free

23 environments, with little work having been conducted with changing landscapes or topography.

24 Here, the impact of spatial heterogeneity on the collective motion exhibited by marching desert locust nymphs was studied under controlled lab conditions. Our experimental circular arenas, incorporating a funnel-like narrowing followed by re-widening, did not constitute a major barrier to the locusts; but, rather, mimicked a changing topography in the natural environment. We examined its effects on macroscopic features of the locust collective behavior, as well as the any changes in their marching kinematics. A major finding was that of the limited extent to which the changing topography affected system-level features of the marching locust group, such as the order parameter and the fraction of walking individuals, despite increased crowding at the funnel. Overall, marching kinematics was also very little affected, suggesting that locust marching bands adjust to the environment, with little effect on the overall dynamics of the group. These findings are in contrast to recent theoretical results predicting that environmental heterogeneities qualitatively alter the dynamics of collectively moving particles; and highlight the crucial role of rapid individual plasticity and adaptability in the dynamics of flocks and swarms. Our study has revealed other important features of the marching behavior of the desert locust in addition to its robustness: the locusts demonstrated both, clear thigmotaxis and a tendency to spread-out and fill the available space.

\section{Keywords}

Collective motion, Swarming, Environmental effects, Topological changes, Spatial heterogeneity, Locust marching bands, Schistocerca gregaria. 


\section{Introduction}

48 Locust swarming constitutes a unique model of animal collective motion (recent review in Ariel 49 and Ayali 2015). Collective motion, being a dominant feature of the behavior of these insects, is 50 also responsible for their notorious reputation as a major pest. Of particular importance is the

51 behavior of the swarms of locust nymphs, comprising millions of insects forming marching 52 bands that may cover vast areas of land, stretching across hills and valleys as far as the eye can see (e.g. Ellis and Ashal 1957; Uvarov 1977; Hunter et al., 2008; our own personal observations

54 of the desert locust in Israel's Negev desert, spring 2013).

Different aspects of locust motion, the interactions between conspecifics and their impact on the build-up and maintenance of coordinated movement, have been studied using controlled laboratory experiments (e.g. Ellis 1951; Buhl et al. 2006; Bazazi et al. 2012; Ariel and Ayali 2015, and references therein). In recent years, advanced tracking algorithms have been utilized to monitor up to several dozen animals at a time at high spatio-temporal resolution for later off-line analysis (e.g, Buhl et al. 2006; Ariel et al. 2014). However, those studies were limited to homogeneous, obstacle-free environments, in order to facilitate emergence of the locust collective motion. Such an environment clearly differs from that encountered by the marching locust swarm (or by any other animals demonstrating mass terrestrial movement) in natural conditions, which may feature a complex terrain, vegetation etc., obscuring and obstructing the path of the locusts.

The effects of an heterogeneous environment on the ability of large systems of moving animals

67 (or particles) to form collective dynamic patterns have been mostly studied in simulations. Theoretical work, using simplified models of collective motion, has predicted that the effect of spatial heterogeneities on the ability of swarms to organize and form synchronized motion is 
70 highly non-trivial and to some extent non-intuitive. For example, obstacles may significantly

71 change the phase diagram of the system, either disrupting or promoting coherent motion,

72 depending on obstacle density, noise intensity, and other model parameters (Hatzikirou and

73 Deutsch 2008; Chepizhko et al. 2013; Chepizhko and Peruani 2015; Quint and Gopinathan

74 2015). Boundaries can promote the formation of vortices (Duparcmeur et al. 1995; Czirok et al.

75 1996; Szabo et al. 2006; Barbaro et al. 2009; Potiguar et al 2014), induce traffic-lane patterns

76 (Hernandez-Ortiz 2009; Ariel et al. 2013) and introduce heterogeneity in densities (Drocco et al.

77 2012; Hernandez-Ortiz 2005). Several theoretical studies have considered the effect of

78 heterogeneous environments on specific animal systems, such as birds (analyzing risks of

79 collision with wind turbines; Croft et al. 2015), fish (predicting the location of Capelin off the

80 Icelandic shores; Barbaro et al. 2009), and living cells (studying the collective cell dynamics of

81 tissue cells in micro-fabricated arenas; Szabo et al. 2006). The study of pedestrian flow through

82 bottlenecks has a rich history of over 100 years (e.g. Helbing 2001; Appert-Rolland et al. 2009).

83 Some of the basic phenomena reported to be associated with a locally reduced mobility or

84 increased density include a drop in flow capacity (depending on bottleneck width), division into

85 free-flowing and congested areas, appearance of shock-like waves, and more. Ample research

86 has also been conducted on the interactions of swarms of autonomous agents (robots) with

87 obstacles (e.g. Trianni et al. 2006) and of virtual particles, within the scope of swarm

88 optimization methods (e.g. Shklarsh et al. 2011).

89 In contrast to the theoretical studies discussed above, relatively few experimental reports are 90 available on the macroscopic dynamics of collectively moving organisms within an 91 heterogeneous environment. These include microbial swarms interacting within complex 92 substrates (Tuson and Weibel 2013), tissue cells in micro-fabricated arenas (motivated by the 
93 highly heterogeneous media that form the extracellular matrix; e.g. Friedl and Broecker 2004;

94 Szabo et al 2006), and pedestrians at bottlenecks, demonstrating a continuous reduction in flow 95 capacity following a reduction in bottleneck width (Hoogendoorn and Daamen 2005; Appert-

96 Rolland et al. 2009). Experiments using different species of ants have shown that topology can

97 have varying effects on the collective movement of the different species. For example, it has

98 been shown that in Atta cephalotes (Burd 2002), increased density due to a reduction in trail 99 width reduces the mean traveling speed and the flux of ants across a barrier. In contrast, the flow 100 of black garden ants (Lasius niger) does not seem to depend on trail width (Dussutour 2004). To 101 the best of our knowledge, locusts, as well as other animal models of collective motion, have 102 been little studied in this respect. The movement of ants along trails fundamentally differs from 103 that of locusts, as ants seldom turn while walking, being typically either non laden when exiting 104 the nest, or laden when returning to it (Burd 2002, Dussutour 2004). In contrast, the frequency of 105 turning (when changing from a standing to a walking state) has been found to be central in the 106 ability of locusts to form and maintain collective movement (Ariel et al., 2014).

107 This current study sought to investigate locust marching behavior in a changing topography: in 108 our case, a circular arena incorporating a funnel-like narrowing followed by a re-widening. This spatial heterogeneity did not constitute a major barrier to the locusts but, rather, introduced certain simple features that are also found in natural environments, such as a path among large rocks or simply a narrowing gorge. We were interested in examining the effects of such

112 topographical changes on macroscopic features of the locust collective behavior, such as the order parameter, as well as any resultant changes in statistics of the marching behavior of

114 individuals (e.g. speed, walking-bout duration etc.), and in the dynamics of local interactions 
115 between the insects, such as distance between neighboring marching locusts, and how these, in 116 turn, translate to swarm dynamics.

117 One of the key questions regarding the response of the individual to a changing environment is 118 that of the effect of topography on the coordinated movement of the crowd. Specifically, it is not 119 clear whether such a response is universal, i.e., common to all swarms, from single cells to 120 locusts and humans; or, alternatively, it significantly differs across species. The main goal of the 121 current study was to revisit previous experimental and theoretical work and to provide novel 122 insights into the effect of a specific type of heterogeneous environment on the collective 123 dynamics of marching locusts. In particular, we sought to unravel the mechanisms by which 124 individual locusts alter their kinematic parameters in order to adjust to the changing environment 125 and diminish its effect on the swarm.

\section{Materials and Methods}

127 Animals

128 Desert locusts, Schistocerca gregaria (Forskål), were obtained from our colony at the 129 Department of Zoology, Tel Aviv University, Israel. The locusts, approaching the gregarious 130 phase, were reared over many generations in 60-liter metal cages at a density of 100-160 animals 131 per cage, under a controlled temperature of $30^{\square} \mathrm{C}$ and $35-60 \%$ humidity, and a $12 \mathrm{D}: 12 \mathrm{~L}$ cycle. 132 Additional radiant heat was provided by $25 \mathrm{~W}$ incandescent electric bulbs during daytime to 133 reach a final day temperature of $35-37^{\llbracket} \mathrm{C}$. The locusts were fed daily with wheat seedlings and 134 dry oats. All experiments were performed on nymphs of the final (Vth) nymphal-instar (3-4 cm 135 in length and $\sim 0.5 \mathrm{~cm}$ in width). 
137 We conducted a series of experiments in each of which a group of nymphs was allowed to move 138 freely in a circular arena. The basic experimental arena was composed of a flat blue Perspex 139 sheet circumscribed by an outer flexible blue plastic wall $(60 \mathrm{~cm}$ diameter $\times 55 \mathrm{~cm} \mathrm{high})$. An 140 inner circular wall made of similar plastic (diameter $30 \mathrm{~cm}$ ) was inserted to create a ring shaped 141 sphere (Fig. 1A). The different experimental conditions were achieved by either leaving the inner 142 circle in the middle (no displacement), or moving it by 5 or $10 \mathrm{~cm}$ sideways towards the outer 143 wall (creating a funnel 10 or $5 \mathrm{~cm}$ wide, respectively; see Fig. 1B). The lower $10 \mathrm{~cm}$ of the 144 arena walls were thinly coated with Fluon (Whitford Plastics Ltd, Runcorn, UK) to prevent the 145 nymphs from climbing. The arena was placed in our temperature-controlled room $\left(30^{\circ} \mathrm{C}\right)$ and lit 146 from above by a $100 \mathrm{~W}$ ring bulb. The propensity of locusts to swarm in an experimental arena is 147 highly sensitive to the number of animals introduced into it (Buhl et al. 2006). Hence, fifty 148 nymphs were introduced into the arena in each experiment, which is a sufficiently high density to 149 facilitate the formation of synchronized movement (Ellis 1951; Buhl et al. 2006; 2011; Ariel et 150 al. 2014), and also approximates the range of densities observed in the field (Ayali et al. 151 unpublished). The locusts were continuously monitored and their movement recorded, using a 152 Sony HDR-XR550E digital camera with a 30 fps rate, for later off-line analysis. Using a customdesigned continuous multiple-target tracking method (Ariel et al. 2014, Fig. 1C), we 154 simultaneously tracked the movement of all individuals at high spatio-temporal resolution (full 155 HD allowed a detailed analysis of the behavior of each individual). We conducted overall 15 experiments, 5 under each experimental condition (inner circle displacement of 0,5 , or $10 \mathrm{~cm}$ ). 157 Each experiment lasted approximately $4 \mathrm{hr}$, from which the last 200,000 frames were selected 158 (ca. $2 \mathrm{hr}$ ), for consistency between experiments and to ensure that we had captured robust locust 159 marching. These were then divided into one hour blocks, providing ample data for statistics. A 
16010 minute period (which is several times longer than the typical resting or walking duration) at

161 the end of each block was discarded to ensure that the blocks were independent of one another.

162 Division into a smaller number of blocks did not qualitatively change the results. Hence, we

163 were left with two sections from each of the five experiments, under each of three experimental

164 condition, totaling 30 experimental sections for further comparative analysis $(\mathrm{n}=10$ for each 165 experimental condition).

166

167

\section{Analysis of behavior}

168

169

170

171

172

173

174

175

176

177

All data analysis was performed in the MATLAB environment (MathWorks, Natick, Massachusetts, USA). To minimize bias wherever possible, blinded methods were used to analyze data. Following smoothing of trajectories as detailed in Ariel et al. (2014), specific attributes of the system and the individual locusts were defined and analyzed as follows.

Identification of walking bouts: An animal was classified as walking if it was moving at a speed greater than $2 \mathrm{~mm} / \mathrm{sec}$ for at least 11 frames. We denote by $w_{k}(t)=0,1$ or -1 if at time $t$ animal $k$ is standing, walking in the counter clockwise (CCW) direction, or walking in the clockwise (CW) direction, respectively. We denote by $f(t)$ the fraction of moving animals at time $t$,

where $N$ is the number of animals in the arena. Thus, $0 \leq f(t) \leq 1$, where $f(t)=0$ implies that

179 all animals are standing, while $f(t)=1$ implies that they are all walking. 
180 For a given animal, the time series $w_{k}(t)$ can be divided into periods of walking and resting, i.e.

181 continuous segments in which either $w_{k}(t)=1$ or -1 . Accordingly, we consider the distribution

182 of walking and resting durations, denoted $t_{\text {walk }}$ and $t_{\text {rest }}$, respectively.

183 Walking speed: The speed of animal $k$ at time $t$ is denoted $v_{k}(t)$.

184 The order parameter: To quantify order and synchronization in the system, we defined the 185 instantaneous order parameter as the average direction $(\mathrm{CW}$ or $\mathrm{CCW})$ of walking animals at time $186 t$. More precisely,

$$
\phi(t)=\frac{1}{N f(t)} \sum_{k} w_{k}(t)
$$

When no animals are moving, the system does not have a preferred direction and $\phi(t)=0$. Thus,

$|\phi|$ is a measure of the level of coordination within the swarm; a value of $|\phi|$ close to zero indicates that the swarm is disorganized, with animals moving in random directions; while a value close to one indicates a highly synchronized state with all directions aligned. The sign of

192 the order parameter indicates the direction of the swarm: positive $\phi$ refers to CCW motion, while negative $\phi$ indicates $\mathrm{CW}$ motion. Note that $\phi$ here differs from the order parameter used by many authors, since it depends only on walking animals.

Number of neighbors: For each frame, we collected all the locations of all locusts and calculated the number of conspecifics up to a distance of $6 \mathrm{~cm}$ from each focal animal

197 (corresponding to circa two locust-body-lengths). The number of neighbors of animal $k$ at time $198 t$ is denoted $n_{k}(t)$. 
199 Number of close moving neighbors: Similar to the number of neighbors, ignoring standing 200 animals. In other words, the number of moving neighbors, $n_{\text {move }, k}(t)$, defined only if animal $k$ is 201 moving at time $t$, is the number of conspecifics walking within a distance of $6 \mathrm{~cm}$ from animal $202 k$. Both the number of neighbors and the number of moving neighbors describe the visual 203 stimulus of an animal and have been previously shown to affect the propensity of animals to start 204 moving (Ariel et al. 2014).

Significance tests: In the comparison of the different arena types the p-Values (in parenthesis) report the probability that the means of all three arenas are the same (an ANOVA analysis calculated using Matlab's One-way analysis of variance function). In the comparison of the arena halves the p-Values (in parenthesis) report the probability that the means obtained from the two halves are the same.

Correlation coefficients: The correlation coefficient, $\rho$, where noted (between any pair of the measurements described above), is the Pearson coefficient using all data points. Correlations were considered as significant for $|\rho|>0.3$, which, for $n=30$ corresponds to a (one-sided) Pvalue of 0.05 or lower.

Heat map representations: An image of the arena was divided into $248 * 248$ pixels. For each pixel, we summed all the times that the center of a locust appeared in it (within one experiment).

216 In order to account for differences between the experiments in terms of overall density, we 217 summed the values from all the pixels together and divided the value of each pixel by that sum. 218 In other words, each pixel now had a value representing the fraction of the animals in that experiment. We visualized this by representing each pixel, according to its value, with a specific color. 
221 Because trajectories are smoothed, they are not as accurate as the raw resolution allows (the error

222 is 3-5 pixels, corresponding to about $1 \mathrm{~mm}$ ). Hence, the reduced resolution used for heat-maps 223 (and only for heat maps) allows convenient representation without any significant loss of 224 accuracy.

225 Heat map of movement: Similar to the heat map, with only moving animals taken into account. 


\section{Results}

229 Changing topography does not affect the global parameters of the locust collective motion

230 The order parameter, $\phi(t)$, is an accepted measure of synchronization in a system (Buhl 2006;

231 Ariel and Ayali 2015). Figure 2A presents two examples of the evolution of the order parameter

232 and the overall fraction of walking locusts, $f(t)$, during a 50 min time section in our

233 experiments. In accordance with these examples, when plotting the average of $|\phi|$ against $f$ in all

234 experiments, they were consistently found to be highly correlated, confirming previous findings

235 regarding the increased tendency of animals to join their conspecifics as the number of walkers

236 increases (Ariel et al. 2014). Neither this correlation nor the actual magnitude of $\phi$ and $f$ were

237 affected by the topography of the arena, i.e. they were independent of the displacement of the

238 inner circle, $d$ (Figs. 2C1 and 2C2, p-Values 0.57 and 0.53 , respectively).

239 Other attributes of the overall kinematics of the marching locusts were also not affected by the

240 arena topography (Fig. 3). These include the average speed of all locusts in the arena (Fig. 3A1, 241 p-Value 0.99), and the duration of a locust's walking or pausing bout (Figs. 3B1 and 2B2, p-

242 Values 0.4 and 0.41). This is not surprising, as many of the marching kinematics showed a 243 significant correlation with $\phi$ and with $f$, again irrespective of the arena topography (see for 244 example a plot of speed vs. $f$, Fig. 3A2). No differences in density-related parameters, such as the 245 number of locusts or of marching locusts only, within a short range from a focal locust were 246 found (Figs. 3C1 and 3C2, p-Values 0.55 and 0.19).

247 Effect of changes in topography as reflected by comparing different parts of the arenas 
248 In contrast to the above findings, a comparative investigation of the local environments within

249 the different experimental arenas (the wide vs. narrow half or, alternatively, the narrowing vs. re-

250 widening section; Fig. 1B) revealed clear effects of the changing topography. Each arena was

251 divided into two equal-area halves. In the case of the symmetrical condition this resulted in two

252 similar-interchangeable halves. In the asymmetrical-heterogeneous conditions, one section

253 contained the funnel and the other section constituted the wider area (Fig. 1B; left and right

254 halves respectively). The duration of a pausing bout showed a clear dependency on the type of

255 experimental arena when comparing the arena sections containing the funnel, i.e. the pause

256 duration was shorter as the funnel became narrower (Fig. 4A, p-Value 0.02). However, although

257 less pronounced, the correlation was opposite in direction when comparing the opposite halves

258 among the different arenas (i.e. longer pause durations in the wider parts of the asymmetric

259 arenas; Fig 4B). In contrast to the clear dependence of rest durations on the arena topography,

260 analysis of the effect on walking durations gave inconclusive results ( $p$-Values 0.18 and 0.21 for

261 the left and right halves). This is in accordance with our previous finding that locusts modulate

262 their rest durations rather than their walking durations (reported previously for a homogeneous

263 environment; Ariel et al. 2014).

264 Focusing on the heterogeneous-asymmetric arenas only, we further compared the kinematics of

265 the locust marching, measured in the arena-half containing the funnel, to those in the arena-half

266 characterized by the wider path (see Fig 5A1). The Most pronounced differences were those

267 found in the arena resulting from the largest displacement (narrowest funnel; the right-hand

268 topography illustrated in Fig. 1B) in respect to the locusts' average walking speed (Fig. 5A2, p-

269 Value $<10^{-8}$ ), and the distribution of the durations of the walking and pausing bouts (Figs. 5A3,

$2705 \mathrm{~A} 4 ; \mathrm{p}$-Values $<10^{-7}$ ). As can be seen, around the funnel the locusts' speed tended to be lower, 
271 with rest or stop durations shorter, but walking durations longer. Interestingly, only a few

272 differences in kinematic parameters were found when comparing the section of the arena

273 containing the narrowing part of the funnel with that containing the widening section (Fig. 5B1).

274 Only the mean speed of the locusts was found to differ (i.e. somewhat increased speed when

275 emerging from the funnel, Fig. 5B2), while the durations of a walking or pausing bout tended to

276 be more similar (Figs. 5B3 and 5B4; p-Values $>0.05$ ).

277 The parameter most affected by the arena topography was found to be the local density of the

278 locusts, as reflected in the number of locusts within a short range from a focal locust. For

279 example, the distribution of close-neighbors count or that of close-moving-neighbors count

280 (comprising only walking insects) was very different in the two halves of the arena formed by a

$28110 \mathrm{~cm}$ displacement (Fig. 6A, p-Value $<10^{-8}$ ). This suggests that the marching locusts do not

282 maintain constant distances from one another, but, rather, tend to temporarily crowd together in

283 response to a narrowing environment. Furthermore, the increase in neighbors-count was found to

284 be positively correlated with the displacement in the arena half containing the funnel, i.e., 285 negatively with the width of the funnel (Figs. 6B1 and 6B2, p-Values $<10^{-4}$ ). In contrast, in the 286 opposite, wide half of the arena, the neighbors-count decreased with the displacement (Figs. 6C1

287 and $6 \mathrm{C} 2$, p-Value $<10^{-4}$ ), suggesting a tendency of the locusts to spread out and fill the available 288 space (see also Fig. 7, showing an example of locust trajectories in the re-widening section of the 289 arena).

290 Effect of changes in the topography as reflected in density heat-maps

291 Figure 8A shows three examples (one for each of the three experimental conditions) of a heat 292 map generated by attributing to each pixel in the arena a color related to the cumulative number 
293 of times it was occupied by a locust during the experiment - from black (unoccupied) to white

294 (most frequently visited). Next, we converted the color code to a value between 0 (unoccupied)

295 to 1 (most frequently visited ), and generated a plot of a slice of the mid-wide half of the arena

296 (Fig. 8B). The resultant mean plots from the different experimental conditions were then overlaid

297 to reveal similarities and topography-dependent differences (Fig. 8C). Three clear features of the

298 locust motion in the different topographies could be observed:

1. Under all the experimental conditions most of the marching took place close to the perimeter of the arena (shaded dark in Fig. 8C). This is probably due to a combination of its circular nature and the reported tendency of locusts to minimize changes in their trajectory while actively marching (Ariel et al. 2014). The three plots (different experimental condition) are very similar in this respect.

2. Nonetheless, it is also clear that the locusts were using the entire space available to them (compare plots in the light shaded areas in Fig. 8C, and see also Fig. 7). This is mostly seen in the plot of the moving locusts (Fig. 8C2).

3. Irrespective of the arena topography, or of the space available to the locusts, the level of marching near the inner wall of the arena was the same (compare the peaks within the light shaded areas in Fig. 8C).

\section{Discussion}

312 The principal findings of the current study were somewhat unexpected and even surprising. We

313 refer to the limited extent to which the changing topography affected both, the macroscopic or 314 system-level features of the marching locust group (order parameter and fraction of walking 
315 individuals), and the marching kinematics (average speed, duration of walking or standing bouts)

316 and more (Figs. 2 and 3). Our experimental manipulation involved narrowing the path available

317 to the locusts by a factor of 3 (from $15 \mathrm{~cm}$ in the symmetrical arena to only $5 \mathrm{~cm}$ in the case of

318 the $10 \mathrm{~cm}$ displacement). However, despite a clear increase in local densities, this funnel-like

319 obstacle was insufficient to disrupt the order and collective movement, at least within the tested

320 experimental parameters. These findings serve as a strong indication of the stability and

321 robustness of locust collective behavior and its tendency to adapt in order to maintain the

322 marching activity.

323 It was only when comparing between local sub-environments (the two halves of the experimental

324 arena) that the effects of the funnel-like topography, or the opposite wider space, became evident

325 (Figs. 4-6). A swarm of marching locusts encountering a funnel like structure in their

326 environment could respond in several possible manners. These include changes in the behavior

327 of the individual locusts as well as in the swarm dynamics. The individual locust may slow down

328 or, alternatively, speed up when encountering an obstacle (a wall or a standing conspecific in our

329 case) in order to bypass it. The locusts may increase their density, i.e. reduce the average

330 distance between conspecifics (Fig. 9A), or change the relative position towards one another

331 (aligning or forming queues), and more. Similarly, at the level of the swarm, various responses

332 could be envisioned for a marching swarm exiting a funnel or experiencing a widening of its

333 path. In this case the two major alternative behaviors would be to spread out and fill the available

334 space (Fig. 9B), or to show a kind of memory of their path history as reflected in the shape of the

335 swarm, i.e. to refrain from spreading out when exiting the funnel (Fig. 9C).

336 Our study provided two important insights. The first: that the locusts tended to spread out and fill

337 the space available to them (as in Fig. 9B rather than in Fig. 9C). Hence, there should be a 
338 theoretical and practical scenario in which this behavior will be opposed or limited by the strong

339 and instrumental tendency of gregarious locusts to aggregate (this limit was not reached in the

340 current work, but may appear at higher animal densities). As we have shown, the funnel-like

341 topography and the narrowing of the path resulted in alteration of the marching kinematics, such

342 that both speed and pausing durations were reduced. Our previous findings, suggesting that it is

343 the locusts' rest durations rather than walking durations that are typically modulated in order to

344 adjust the swarm dynamics (Ariel et al. 2014), are thus confirmed, supporting the instrumental

345 role of the intermittent motion (pausing) in the marching dynamics.

346 The second prominent feature demonstrated by the locusts in our experimental arenas was that of

347 their attraction to the inner wall. What might appear to be attraction to the outer wall could be, at

348 least partly, a result of a tendency to maintain the walking trajectory (Ariel et al. 2014). Many

349 animals, insects included, exhibit an attraction to walls while moving in a defined space (see for

350 example cockroaches: Jeanson et al., 2005, or flies: Soibam et al., 2012). The tendency to orient

351 in space by means of mechanical contact is termed "thigmotaxis" (Fraenkel and Gunn 1961).

352 Thigmotactic animals have a higher probability of walking adjacent to walls, although different

353 curvatures of walls may have different effects: concave wall has a higher "attraction level" than a

354 convex one (Creed and Miller, 1990). A tendency to follow walls (or, alternatively, a crest or

355 ridge in the landscape) could potentially be advantageous for swarm maintenance, by way of

356 increasing the likelihood of encountering a conspecific, and could thus be important in the

357 collective behavior (Jeanson et al. 2003).

358 It is important to note that there are probably species-specific differences related to the above

359 features, i.e. the density within the swarm and, mostly, the shape of the swarm (e.g. Uvarov

360 1977; Hunter et al. 2008; Buhl et al. 2011; Ariel and Ayali 2015). Species may also differ in the 
361 landscape- or topography-dependent effects on the behavior of individuals and on the consequent

362 changes in the spatial attributes of the swarm. In accordance with our present findings, $S$.

363 gregaria marching bands under natural conditions have indeed been reported to spread out

364 widely along very large distances, in contrast to the long ribbon-like structures reported for other

365 species (Kennedy 1939, 1951; Uvarov 1977).

366 One should of course also be cautious when extrapolating from the results of laboratory

367 experiments to the natural behavior of locusts in the field. The current study constitutes a first

368 step in exploring the important yet very broad question of the effect of topology on the collective

369 dynamics of marching locusts. Hence, we chose here to concentrate on a relatively

370 straightforward regime of experimental conditions, i.e. a specific number of animals and an arena

371 area that had been found to be favorable for promoting locust marching. In other words, the

372 experimental conditions were chosen so that the only disrupting factor for the marching animals

373 would be the narrowing funnel. The effects of other sources of disruption to the coherent

374 marching (for example, over- or under-crowding, large or small distances from the walls,

375 different nutrition etc.), may be non-additive and could significantly change some of the

376 conclusions of the current study. Nonetheless, our results show that even under "optimal"

377 marching conditions, a simple topological heterogeneity has an interesting and somewhat

378 unexpected impact on the locust dynamics.

379 Finally, we emphasize our findings regarding the capacity of locusts to adapt their response to 380 their surroundings (the swarm dynamics) in order to compensate for barriers and bottlenecks in

381 their path. Such behavioral strategies can explain the non-intuitive independence of the order

382 parameter from the funnel width. This experimental finding contradicts several previous

383 theoretical predictions, which suggested that environmental heterogeneities can qualitatively 
384 alter the dynamics of collectively moving particles in a complex way - decreasing the flux

385 through the bottleneck, promoting particular swarming patterns, and even changing the phase-

386 behavior of the system (see Introduction for details). Our results show that, at least under the

387 density regimes tested, persistent and stable dynamics can be promoted by means of individual

388 adaptability. For example, whereas Shklarsh et al. (2011) demonstrated that individual

389 adaptability can enhance search efficiency in swarm optimization methods, our results suggest

390 that adaptability can lead to an opposite effect and increase robustness and resilience. Future

391 experimental and theoretical studies should thus investigate organisms and models with

392 performance-adaptable interactions.

\section{References}

Appert-Rolland C, Chevoir F, Gondret P, Lassarre S, Lebacque JP, Schreckenberg M (Eds.) (2009). Traffic and Granular flow'07. Springer Science \& Business Media.

Ariel G, Ayali A (2015). Locust Collective Motion and Its Modeling. PLoS Comp. Biol. 11(12): e1004522.

Ariel G, Ophir Y, Levi S, Ben-Jacob E, Ayali A (2014). Individual pause-and-go motion is instrumental to the formation and maintenance of swarms of marching locust nymphs. PloS One, 9(7): e101636.

406

Ariel G, Shklarsh A, Kalisman O, Ingham C, Ben-Jacob E (2013). From organized internal traffic to collective navigation of bacterial swarms. New J. Phys., 15(12): 125019.

Barbaro ABT, Taylor K, Trethewey PF, Youseff L, Birnir B (2009). Discrete and continuous models of the dynamics of pelagic fish: application to the capelin, Math. Comput. Sim. 79: 3397. 
407

408

409

410

411

412

413

414

415

416

417

418

419

420

421

422

423

424

425

426

427

428

429

430

Bazazi S, Bartumeus F, Hale JJ, Couzin ID (2012). Intermittent motion in desert locusts: behavioral complexity in simple environments. PLoS Comp. Biol. 8(5): e1002498.

Buhl J, Sumpter DJT, Couzin ID, Hale JJ, Despland E, Miller E, R Simpson SJ (2006). From disorder to order in marching locusts. Science 312(5778): 1402-1406.

Buhl J, Sword GA, Clissold FJ, Simpson SJ (2011). Group structure in locust migratory bands. Behav. Ecol. Sociobiol. 65(2): 265-273.

Burd M, Archer D, Aranwela N, Stradling DJ (2002). Traffic dynamics of the leaf-cutting ant, Atta cephalotes, Am. Nat. 159(3): 283.

Chepizhko O, Peruani F (2015). Active particles in heterogeneous media display new physics, Eur. Phys. J. Special Topics 224(7): 1287.

Chepizhko O, Altmann EG, Peruani, F (2013). Optimal Noise Maximizes Collective Motion in Heterogeneous Media, Phys. Rev. Lett. 110(23): 238101.

Creed Jr RP, Miller JR (1990). Interpreting animal wall-following behavior. Experientia 46(7): 758-761.

Croft S, Budgey R, Pitchford JW, Wood AJ (2015). Obstacle avoidance in social groups: new insights from asynchronous models, J. R. Soc. Interface 12: 20150178.

Czirok A, Ben-Jacob E, Cohen I, Vicsek T (1996). Formation of complex bacterial colonies via self-generated vortices, Phys. Rev. E 54: 1791.

Drocco JA, Reichhardt CJO, Reichhardt C (2012). Bidirectional sorting of flocking particles in the presence of asymmetric barriers, Phys. Rev. E 85: 056102.

Duparcmeur YL, Herrmann H, Troadec JP (1995). Spontaneous Formation of Vortex in a System of Self Motorised Particles, J. Phys. I (France) 5: 1119.

Dussutour A, Fourcassie V, Helbing D, Deneubourg JL (2004). Optimal traffic organization in ants under crowded conditions, Nature 428(6978): 70. 
431

432

433

434

435

436

437

438

439

440

441

442

443

444

445

446

447

448

449

450

451

452

453 455

454 Jeanson R, Blanco S, Fournier R, Deneubourg JL, Fourcassié V, Theraulaz G (2003). A

Ellis PE (1951). The Marching Behaviour of Hoppers of the African Migratory Locust (Locusta migratoria migratorioides R. \& F.) in the Laboratory, Anti-Locust Bull. 7.

Ellis PE, Ashall C (1957). Field studies on diurnal behaviour, movement and aggregation in the Desert Locust (Schistocerca gregaria Forskål), Anti-Locust Bull. 25: 1-94.

Fraenkel GS, Gunn DL (1962). The orientation of animals: Kineses, taxes and compass reactions, Dover, New York.

Friedl P, Broecker EB (2004). The biology of cell locomotion within a three dimensional extracellular matrix, Cell Motil. Life Sci. 57: 41.

Hatzikirou H, Deutsch A (2008). Cellular automata as microscopic models of cell migration in heterogeneous environments, Curr. topics in dev. Biol. 81: 401-434.

Helbing D (2001). Traffic and Related Self-Driven Many-Particle Systems, Rev. Mod. Phys. 73(4): 1067-1141.

Hernandez-Ortiz JP, Stoltz CG, Graham MD (2005). Transport and Collective Dynamics in Suspensions of Confined Swimming Particles, Phys. Rev. Lett. 95: 204501.

Hernandez-Ortiz JP, Underhill PT, Graham MD (2009). Dynamics of confined suspensions of swimming particles, J. Phys.: Condens. Matter 21: 204107.

Hoogendoorn SP, Daamen W (2005). Pedestrian behavior at bottlenecks. Transportation, Science, 39(2): 147-159.

Hunter DM, McCulloch L, Spurgin PA (2008). Aerial detection of nymphal bands of the Australian plague locust (Chortoicetes terminifera (Walker)(Orthoptera: Acrididae), Crop Prot. 27(1): 118-123.

Jeanson R, Rivault C, Deneubourg JL, Blanco S, Fournier R, Jost C, Theraulaz G (2005). Self-organized aggregation in cockroaches, Anim. Behav. 69(1): 169-180. model of animal movements in a bounded space, J. Theor. Biol. 21; 225(4): 443-451. 
456

457

458

459

460

461

462

463

464

465

466

467

468

469

470

471

472

473

474

475

476

477

478

Kennedy JS (1939). The behaviour of the Desert Locust (Schistocerca gregaria (Forsk.))(Orthopt.) in an outbreak centre, Trans. R. Entomol. Soc. Lond. 1:89(10): 385-542.

Kennedy JS (1951). The migration of the desert locust (Schistocerca gregaria Forsk.). I. The behaviour of swarms. II. A theory of long-range migrations, Phil. Trans. R. Soc. London. B 235(625): 163-290.

Potiguar FQ, Farias GA, Ferreira WP (2014). Self-propelled particle transport in regular arrays of rigid asymmetric obstacles, Phys. Rev. E 90: 012307.

Quint DA, Gopinathan A (2015). Topologically induced swarming phase transition on a 2D percolated lattice, Phys. Biol. 12: 046008.

Soibam B, Goldfeder RL, Manson-Bishop C, Gamblin R, Pletcher SD, Shah S, Gunaratne GH, Roman GW (2012). Modeling Drosophila positional preferences in open field arenas with directional persistence and wall attraction. PloS ONE 7(10): e46570.

Shklarsh A, Ariel G, Schneidman E, Ben-Jacob E (2011). Smart swarms of bacteria-inspired agents with performance adaptable interactions, PLoS Comp. Biol. 7(9): e1002177.

Szabo B, Szollosi GJ, Gonci B, Juranyi Zs, Selmeczi D, Vicsek T (2006). Phase transitions in the collective migration of tissue cells: experiment and model. Phys. Rev. E 74: 061908.

Trianni V, Nolfi S, Dorigo M (2006). Cooperative hole avoidance in a swarm-bot, Rob. Aut. Syst. 54(2): 97-103.

Tuson HH, Weibel DB (2013). Bacteria-surface interactions, Soft matter 9(17): 4368-4380.

Uvarov B (1977). Grasshoppers and locusts. A handbook of general acridology. Volume 2. Behaviour, ecology, biogeography, population dynamics. Centre for Overseas Pest Research. 


\section{Figure legends}

480 Figure 1

481 A. A top view of the experimental circular arena. B. The different experimental settings: 482 displacement of the inner circle results in different-width funnel-like structures. The width of the 483 different sections of the arena are noted. C. A snap-shot from a video monitoring of the locusts' 484 collective motion. All nymphs are assigned numbers and their velocities are noted.

485 Figure 2

486 Global parameters of the locust collective motion. A. The correlated changes in the order 487 parameter, $\phi$ (blue), and the overall fraction of walking locusts, $f$ (red), during a 50 min section 488 of two experiments. B. The correlation between the size of the order parameter, $|\phi|$, and the 489 overall fraction of walking locusts, $f$, in all the experiments with the different arenas, as depicted 490 in Fig 1B: symmetrical (red), $5 \mathrm{~cm}$ (blue) and $10 \mathrm{~cm}$ displacement (black). $\mathrm{N}=10$ each. Linear 491 regression is shown in solid lines and the significance of the regression is noted. C. The overall 492 fraction of walking locusts, $f(\mathrm{C} 1)$ and the calculated average order parameter, $|\phi|(\mathrm{C} 2)$ in the 493 different arenas. No arena-type-related differences were found and the non-significant 494 correlation is noted.

495 Figure 3

496 A. No arena-type-related differences were found in average walking speed (A1) However, 497 significant correlations $(\mathrm{P}-\mathrm{value}<0.05)$ were found between the locust average walking speed and 498 the overall fraction of walking locusts (A2) in all three arena types (marked with different 499 colors). B. No arena-type-related differences were found in the average duration of resting (B1) 
500 or walking (B2) bouts. C. No arena-type-related differences were found in the average number of

501 overall neighbors (C1) or walking neighbors (C2) within a short range around a focal locust. The

502 correlation coefficients are noted above each graph.

503 Figure 4

504 A. A clear arena-type-related difference in the average duration of resting (pausing) bouts was

505 found when comparing the arena halves containing the funnel (in the symmetric, 0 displacement

506 arena, one half was selected randomly). B. A similar comparison of the opposite half of the arena

507 yielded a non-significant correlation. The correlation coefficients are noted above each graph.

508 Figure 5

509 A comparison of the distribution of different marching kinematic parameters between the two

510 halves of the arena with a $10 \mathrm{~cm}$ displacement: A. the narrow vs. the wide half, and B. the

511 converging vs. the widening half. In both $\mathbf{A}$ and $\mathbf{B}$, panel 1 shows a schematic sketch (direction

512 of marching is noted in B1); 2. The average walking speed; 3. Durations of resting bouts; and 4.

513 Durations of walking bouts. Colors are matched to the different arena halves as shown in A1 and

514 B1. While all tested parameters differ between the two halves in A, the only noticeable

515 differences in $\mathbf{B}$ are in speed.

516 Figure 6

517 A comparison of locust densities in the narrow and wide halves of the arena. As expected, the

518 number of neighbors (both total as well as walking only) within $6 \mathrm{~cm}$ of a moving focal animal is

519 highly dependent on the displacement (type of arena). A. Distribution of the number of neighbors

520 (left) or moving neighbors (right) in the arena with a $10 \mathrm{~cm}$ displacement (narrowest funnel; 
521 curve colors match the halves of the arena in the enclosed schematic drawing). B. Average

522 number of neighbors in each experiment (narrow half). C. Average number of neighbors in each

523 experiment (wide half). The correlation coefficients are noted above each graph.

524

525

526

527 Figure 7

528 Example trajectories of locusts leaving the funnel (overall 10 nymphs passing within a few

529 seconds through the section marked white in the inset). A tendency of locusts to spread out and

530 fill the available space can be observed.

531 Figure 8

532 Density heat maps and their analysis. A. Example heat maps for all three arena types. White 533 regions denote frequently visited areas. B. Schematic sketch showing a cut through heat map, 534 translated into a graph. Density along the cut is rescaled to values between 0 (no locusts) and 1 535 (maximal observed density). The abscissa denote the location along the section, with the 536 perimeter of the arena on the right and its center on the left. C. Density through similar cuts as in 537 B, averaged over all experiments in each arena type. 1. Density of animals. 2. Density of moving 538 animals only. Shaded areas are explained in the text.

539 Figure 9 
540 Cartoon showing possible swarm behavior in nature. A. Converging into a funnel results in

541 increased density. B. Spreading out of a funnel. C Exiting a funnel while preserving the swarm's

542 shape. See further details in the text (Discussion). 
Figure 1

Fig 1
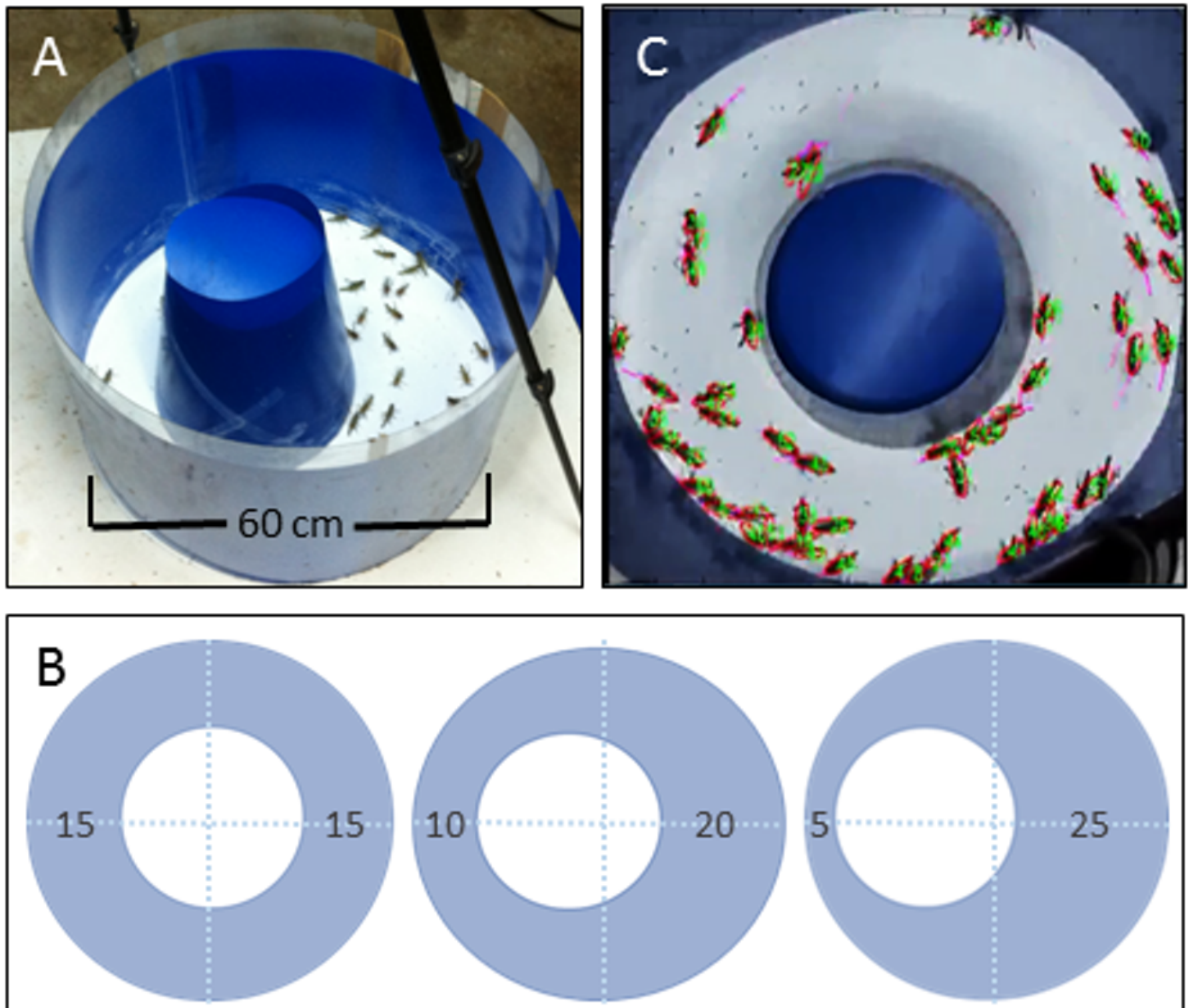

Symmetric $5 \mathrm{~cm}$
displacement no displacement

$10 \mathrm{~cm}$ displacement 
Figure 2

Fig 2
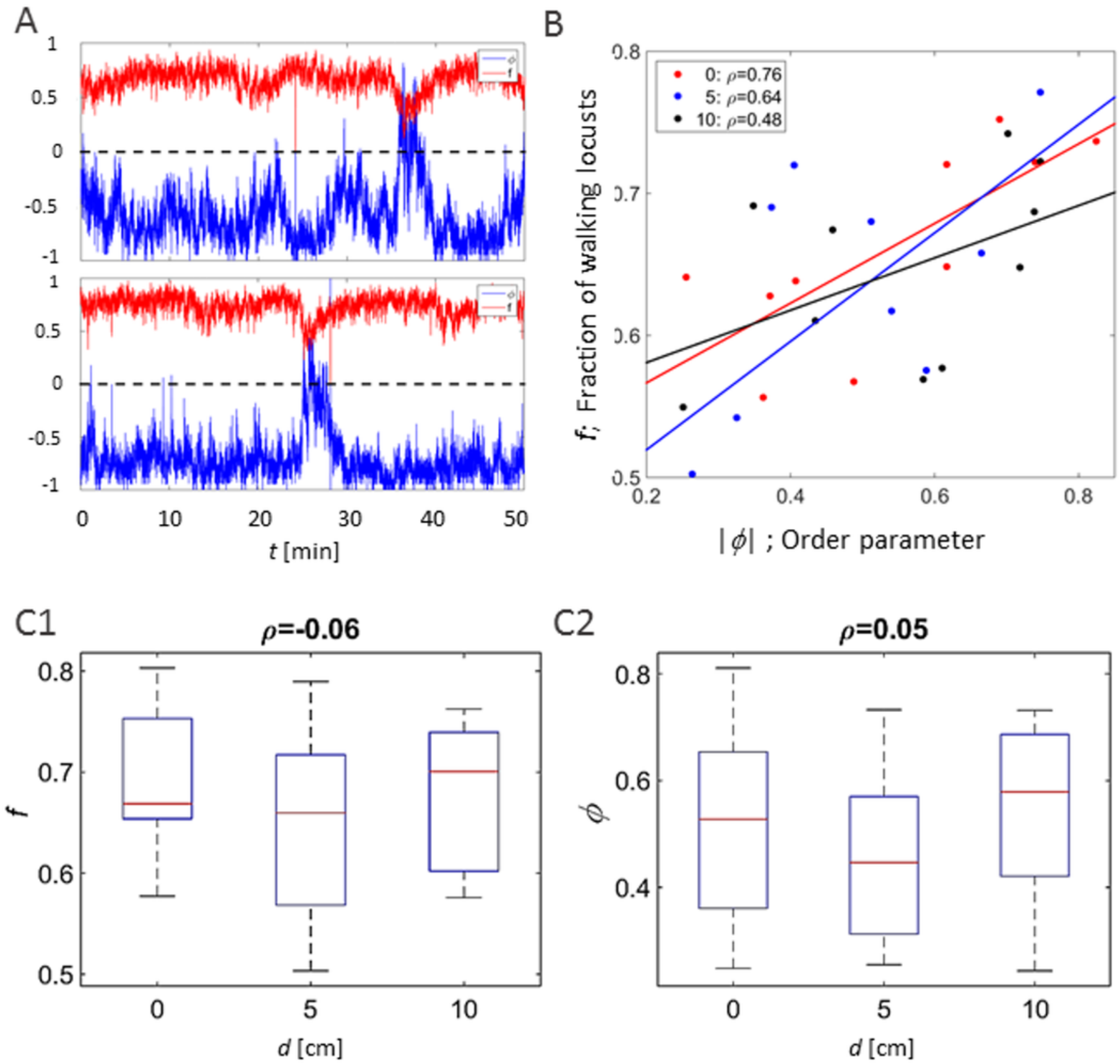

C2

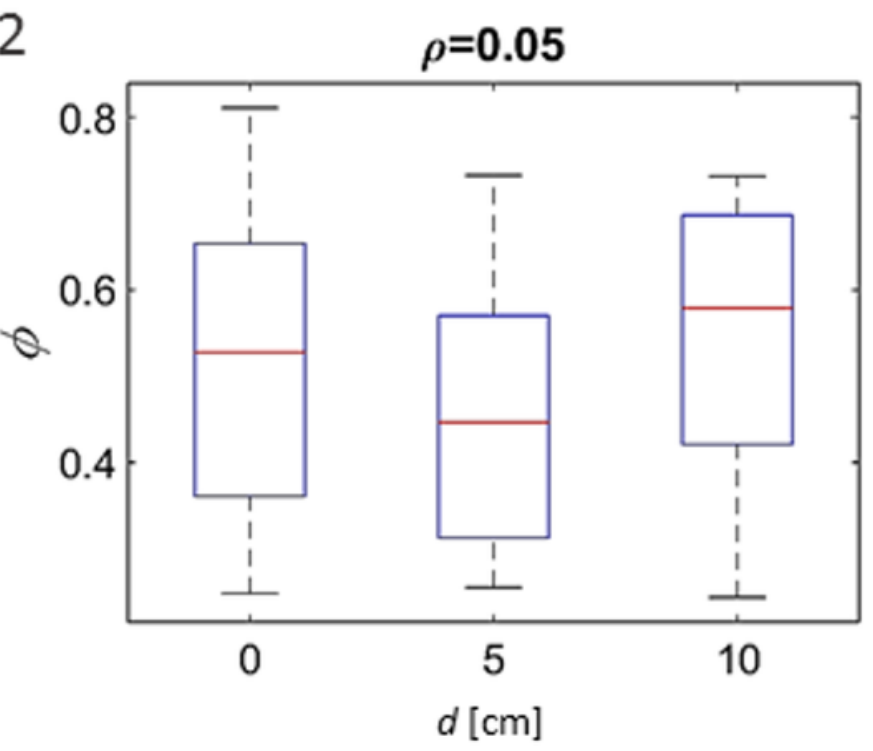


Figure 3

Fig 3 

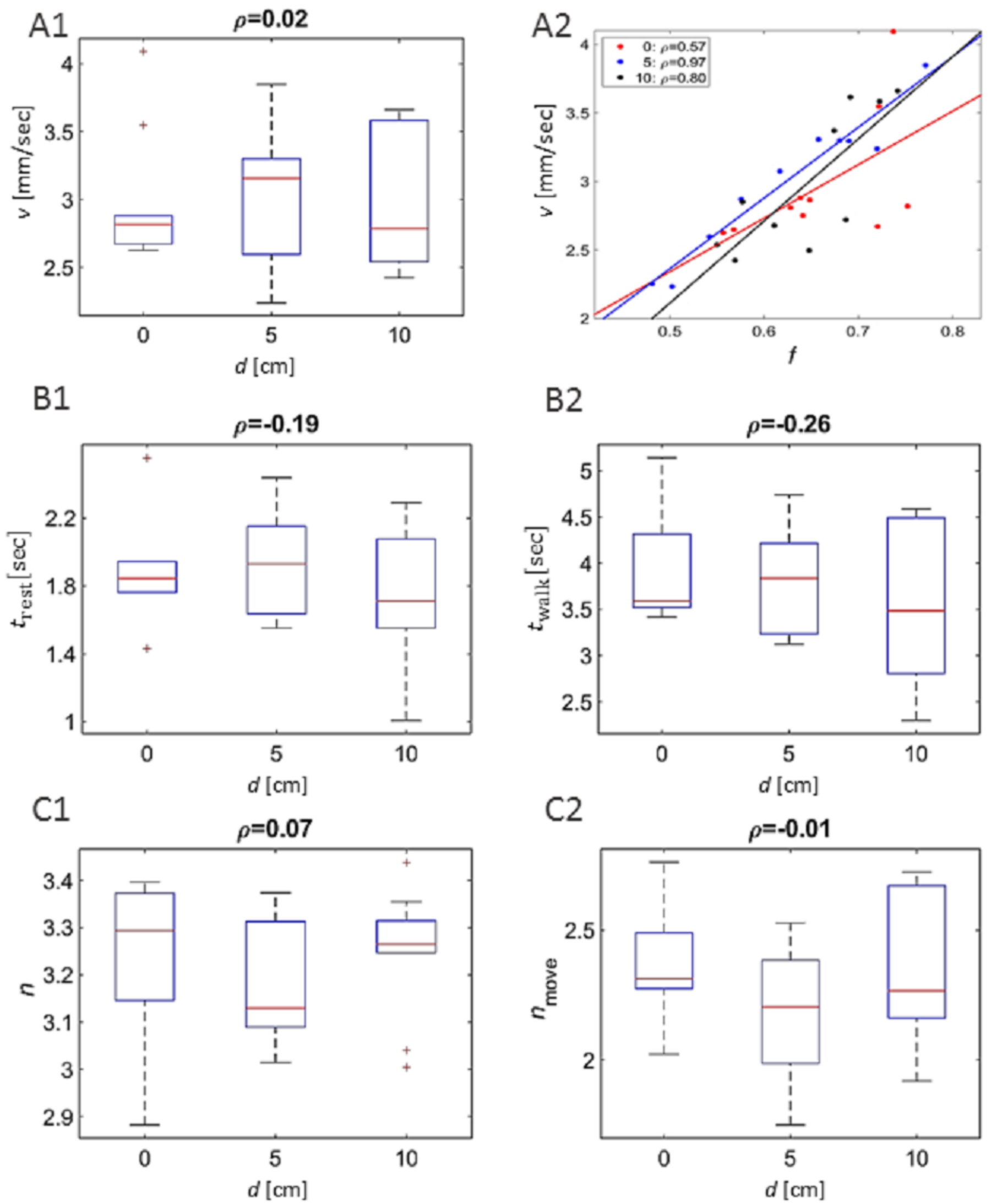
Figure 4

Fig 4
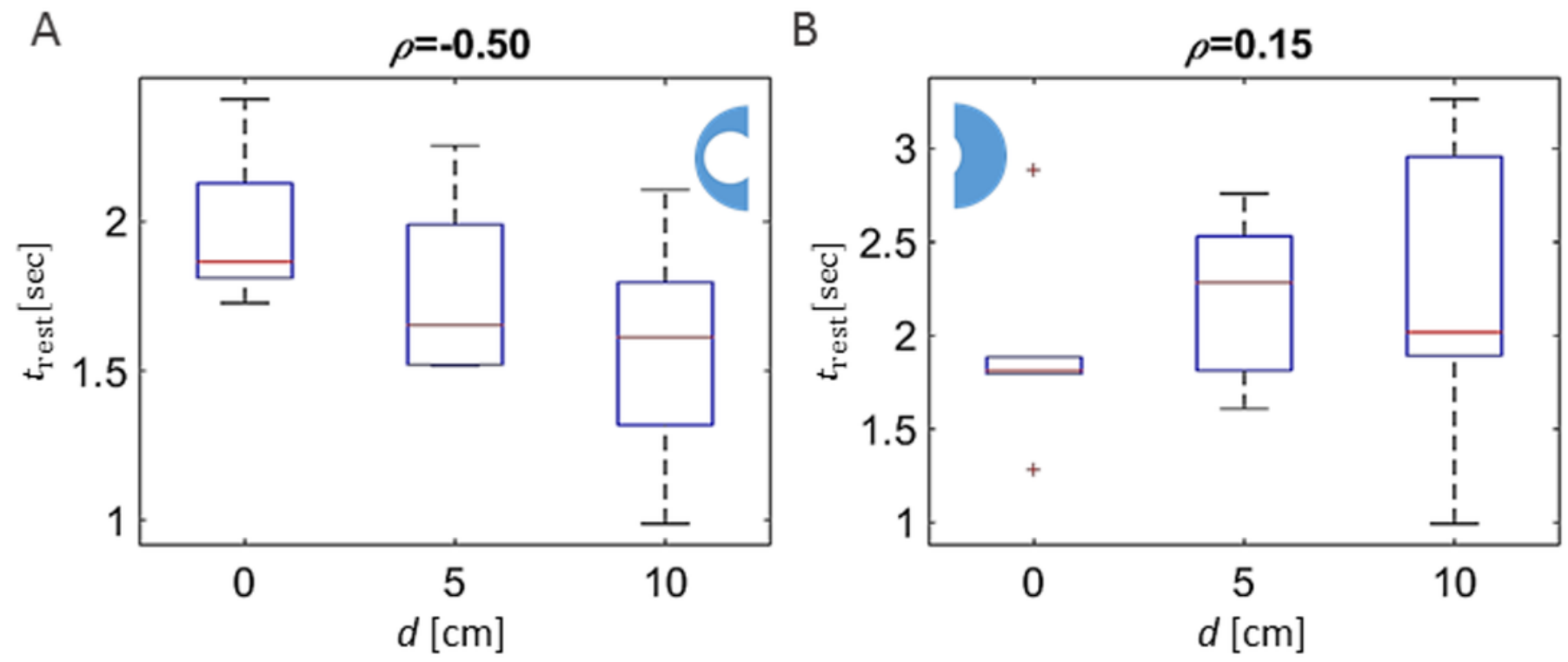
Figure 5

Fig 5 
A1
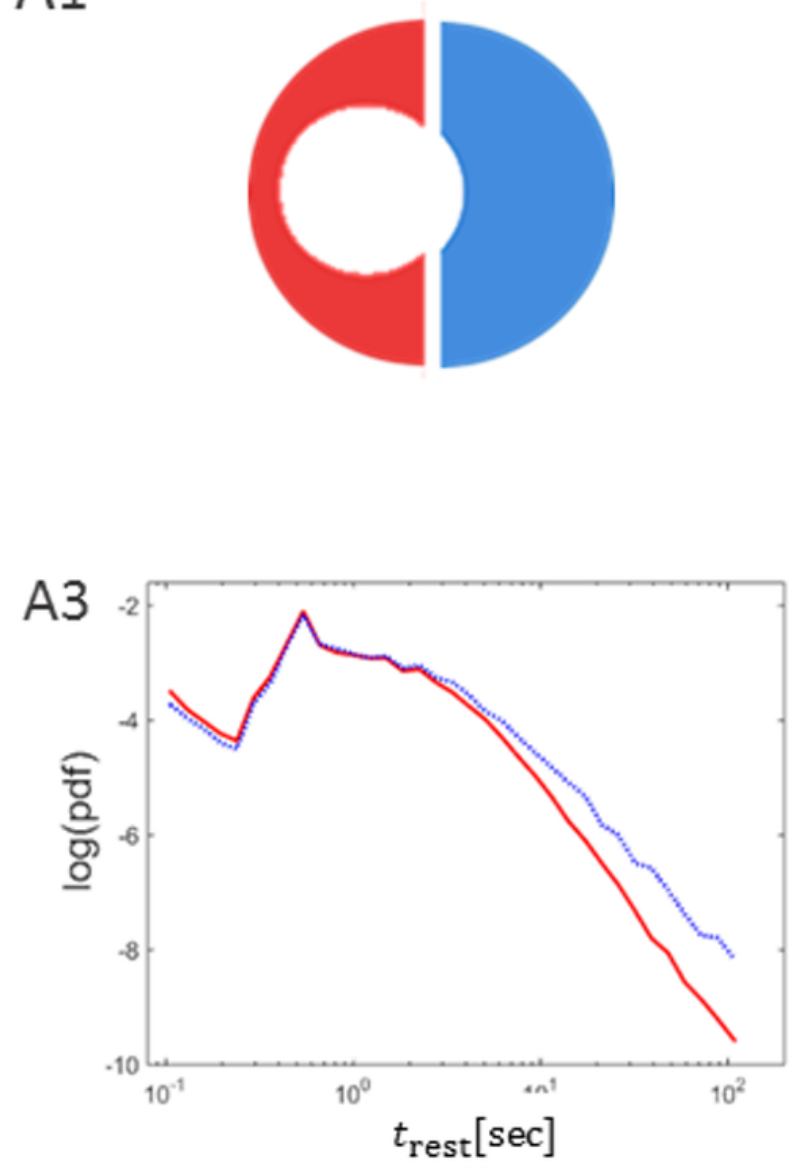

B1

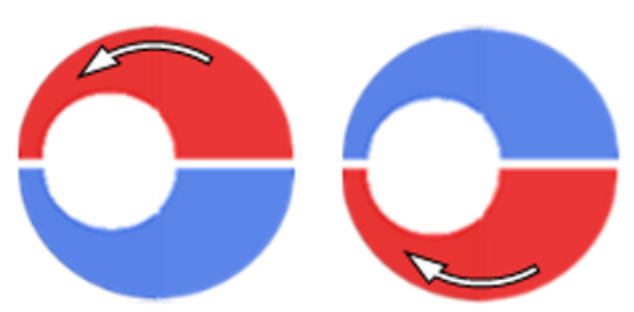

B3

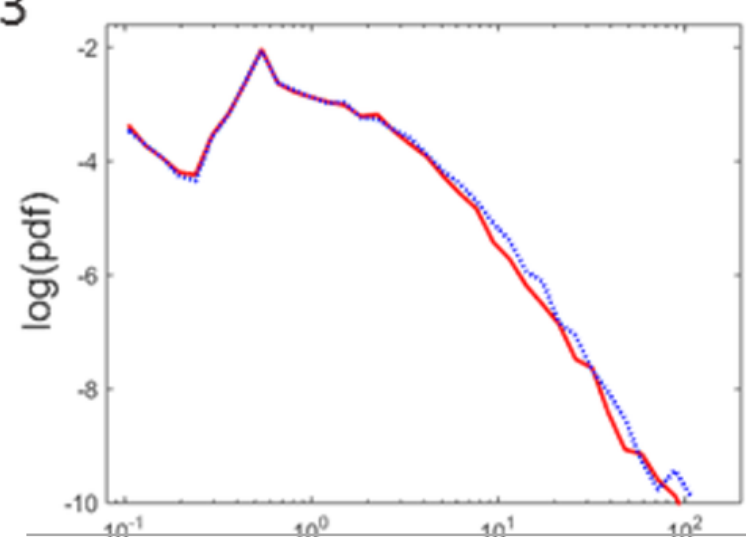

Peer] reviewing PDF | (2016:07:124446:2:0:NEW $29^{10^{2}}$ Oct 2016) $t_{\text {rest }}[\mathrm{sec}]$
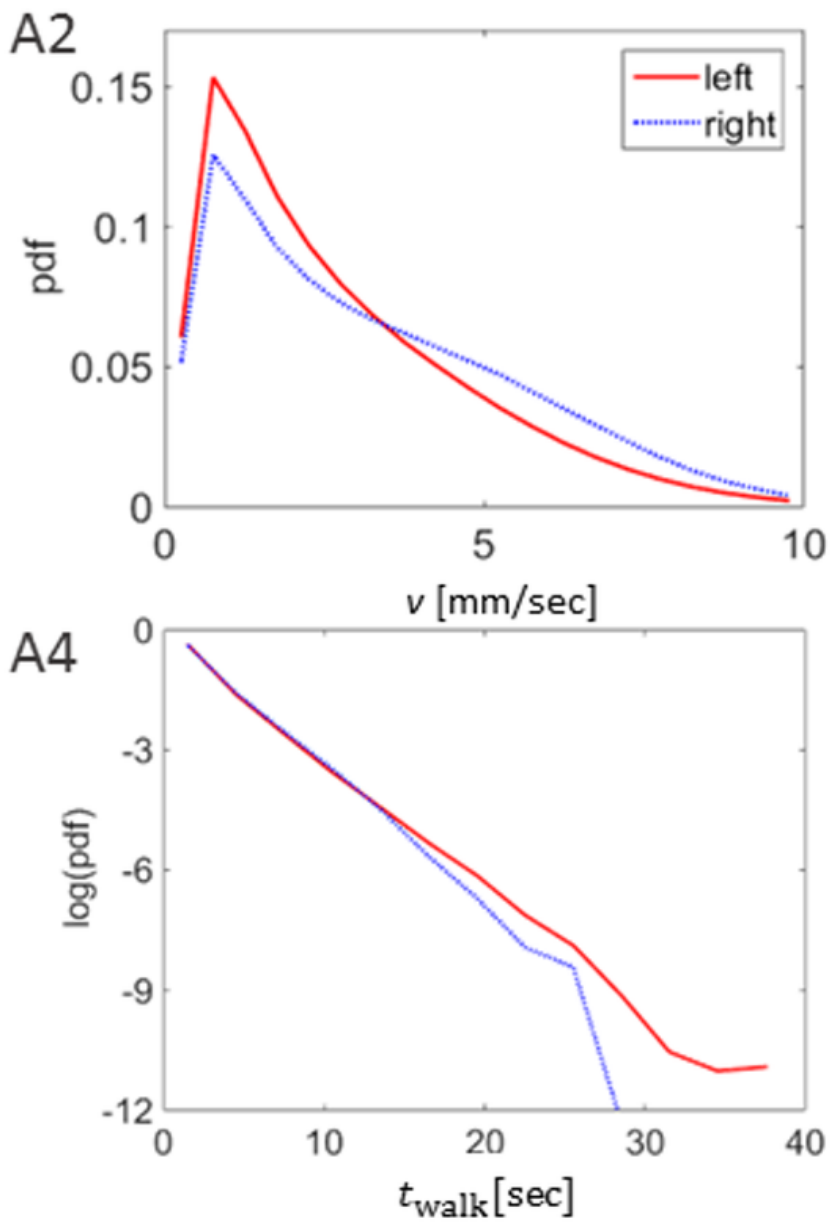

B2

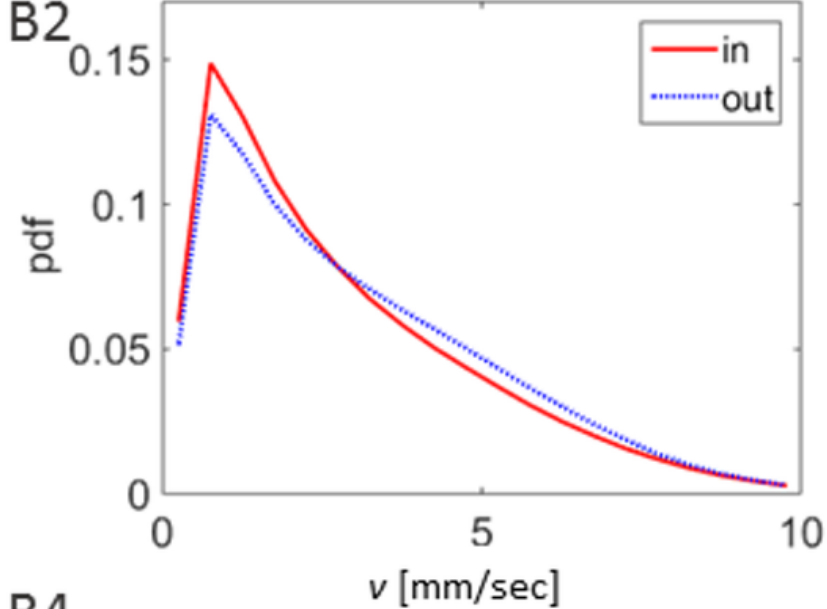

B4

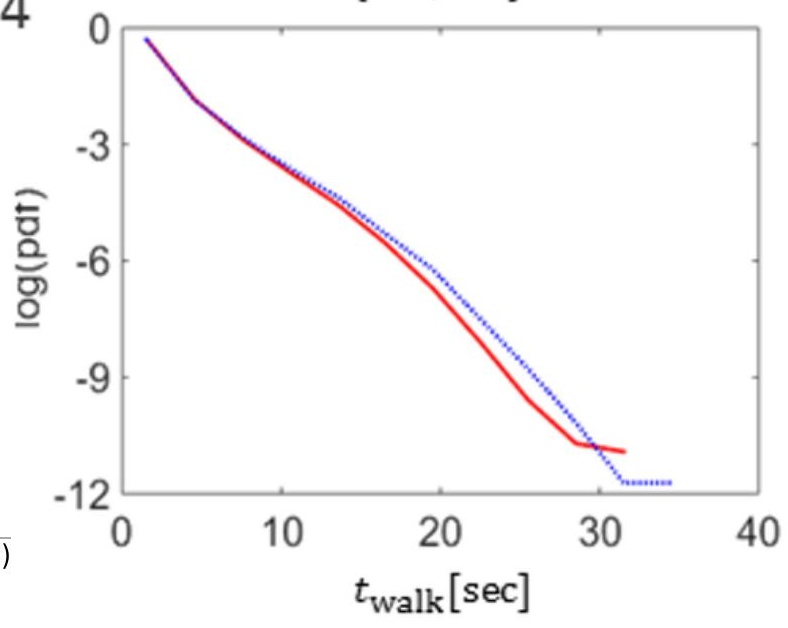


Figure 6

Fig 6 

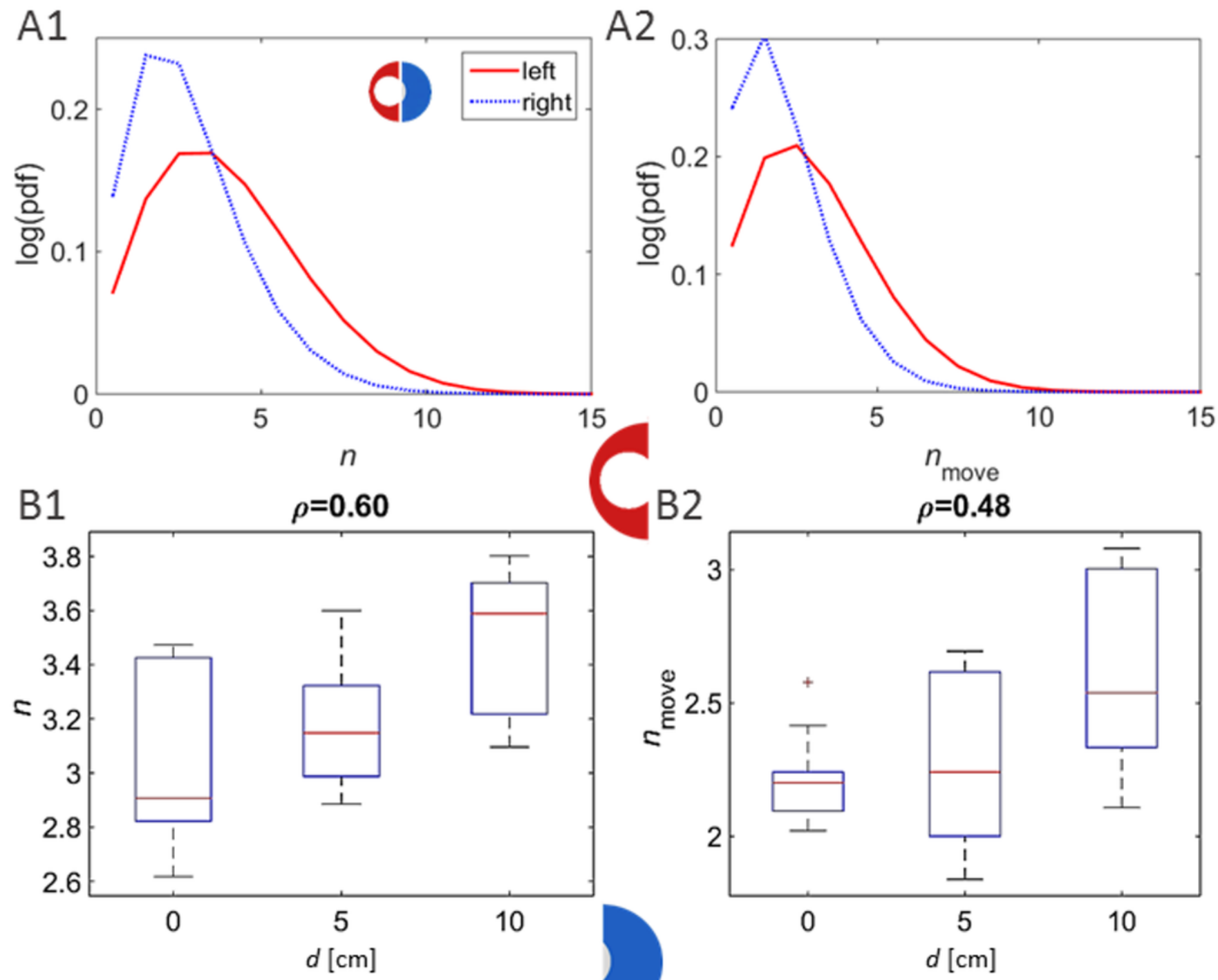

B2
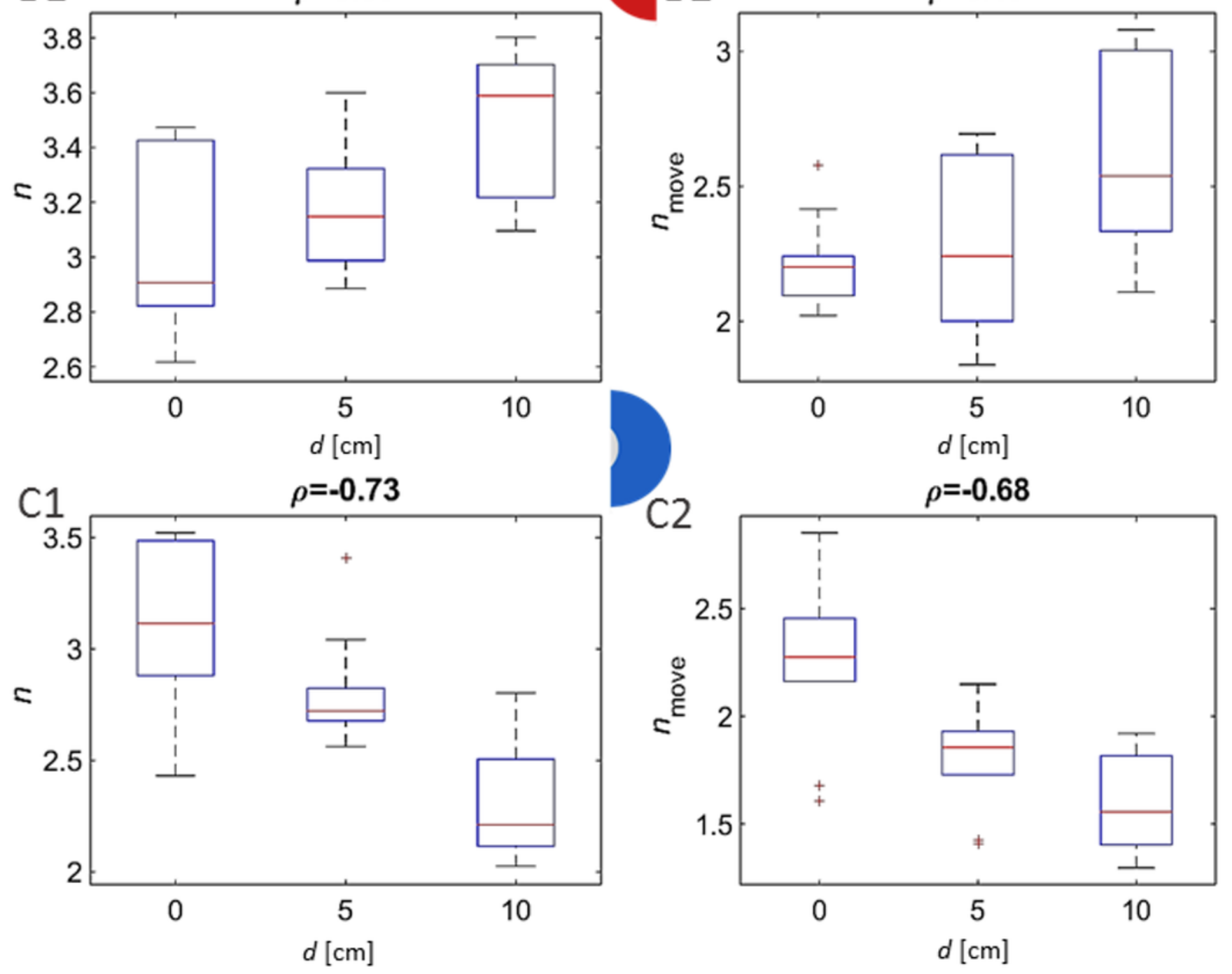
Figure 7

Fig 7

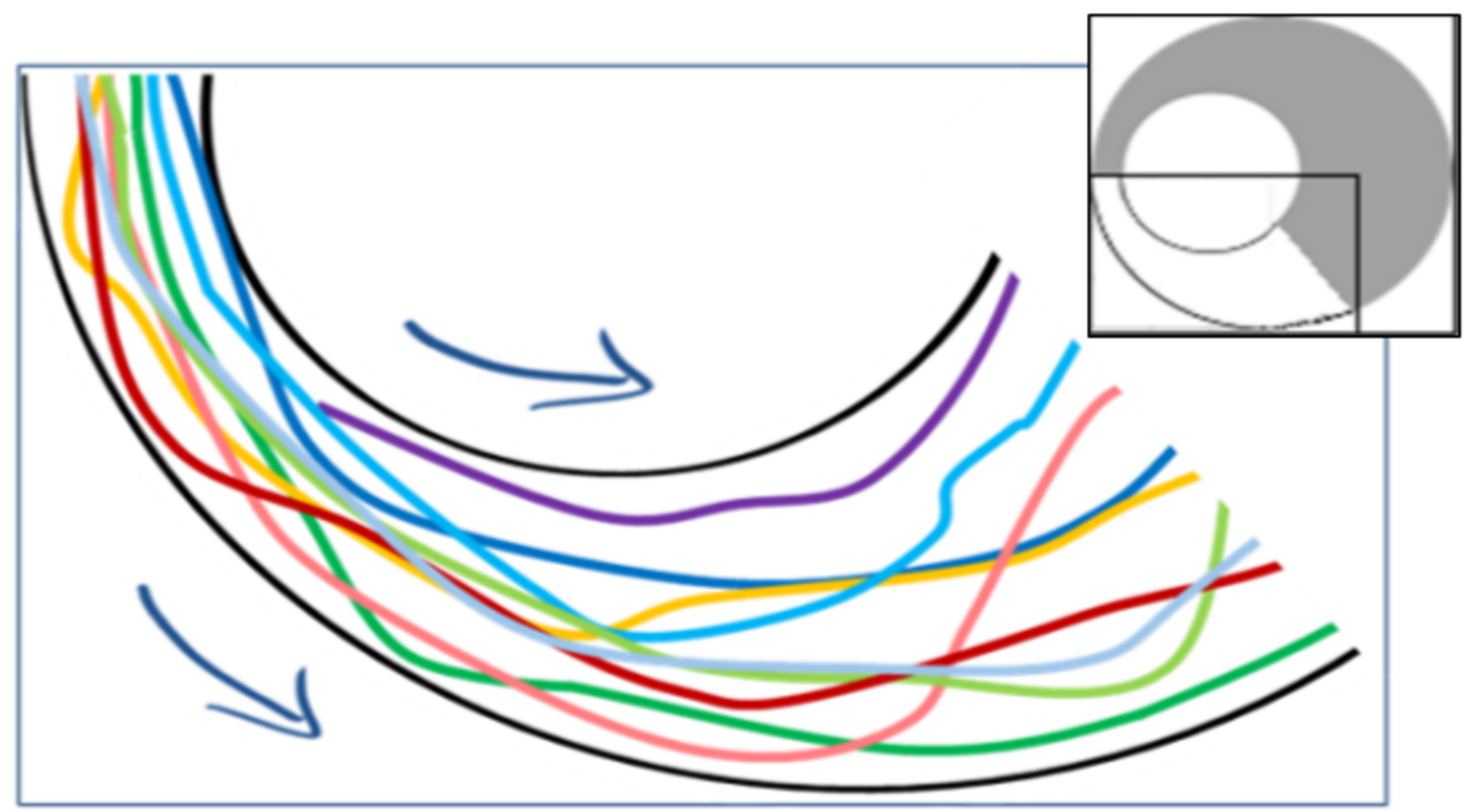


Figure 8

Fig 8
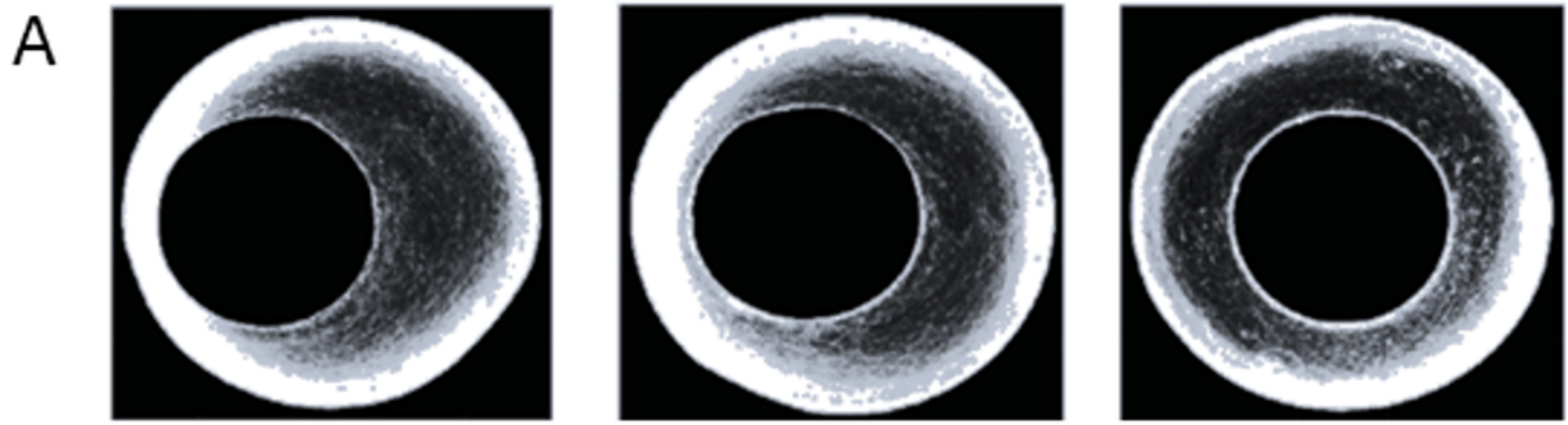

B
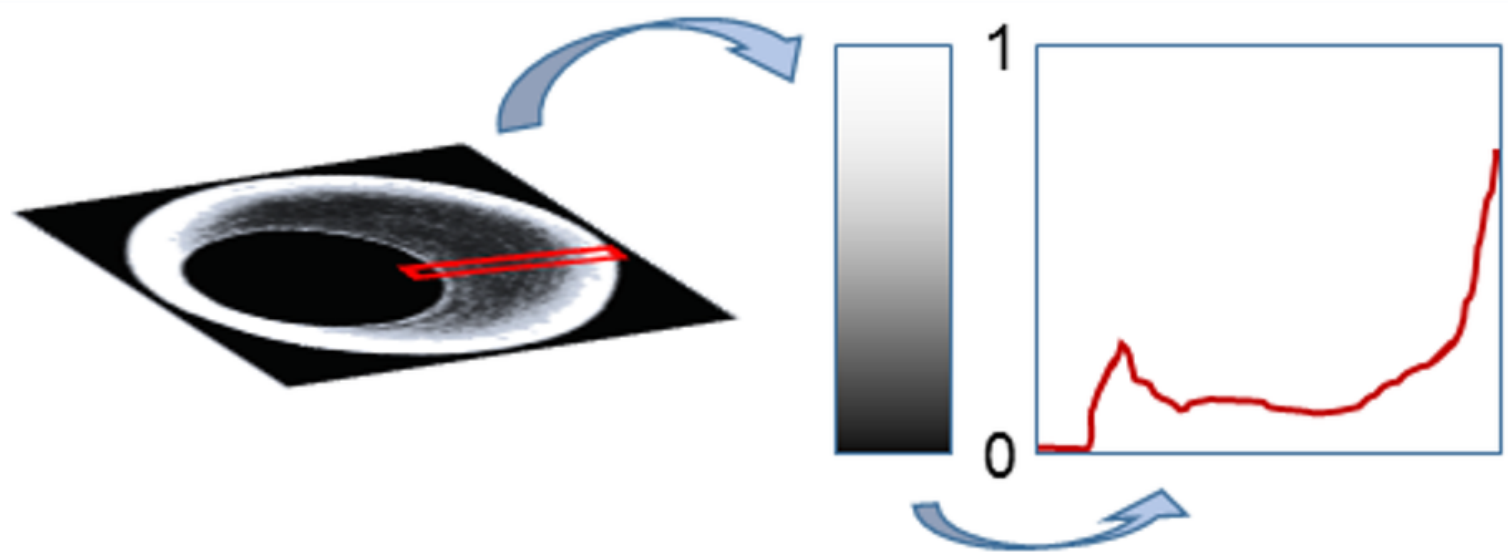

$\mathrm{C} 1$

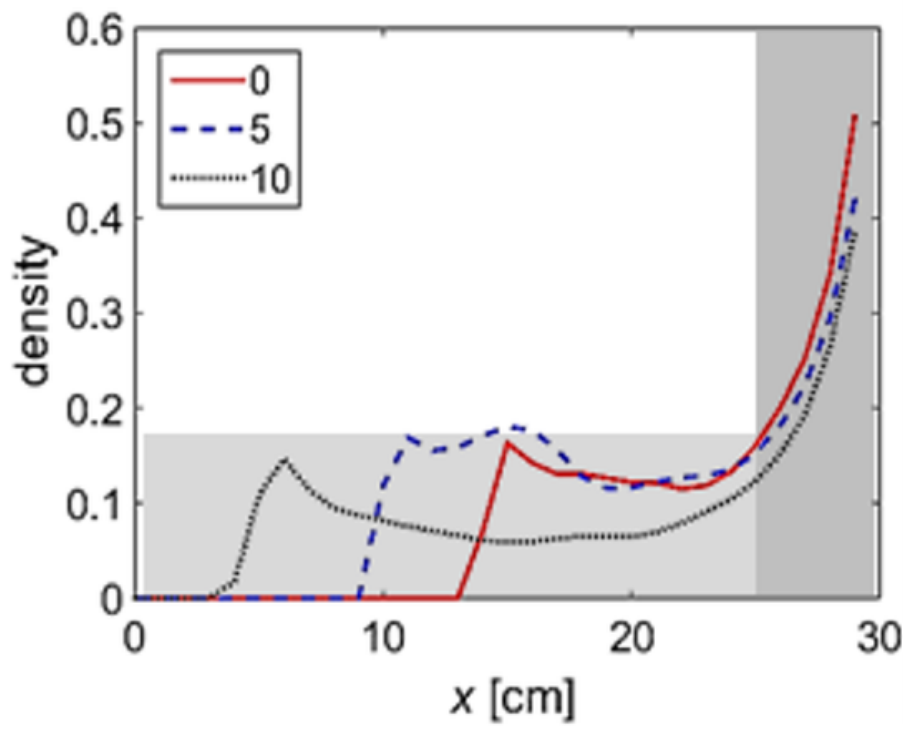

$\mathrm{C} 2$

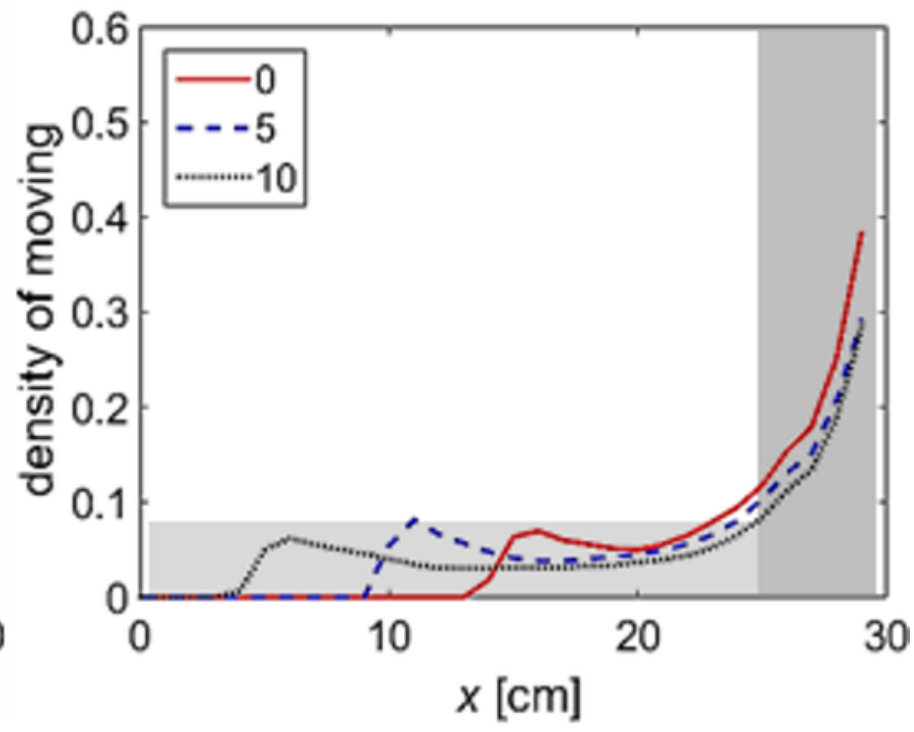


Figure 9

Fig 9

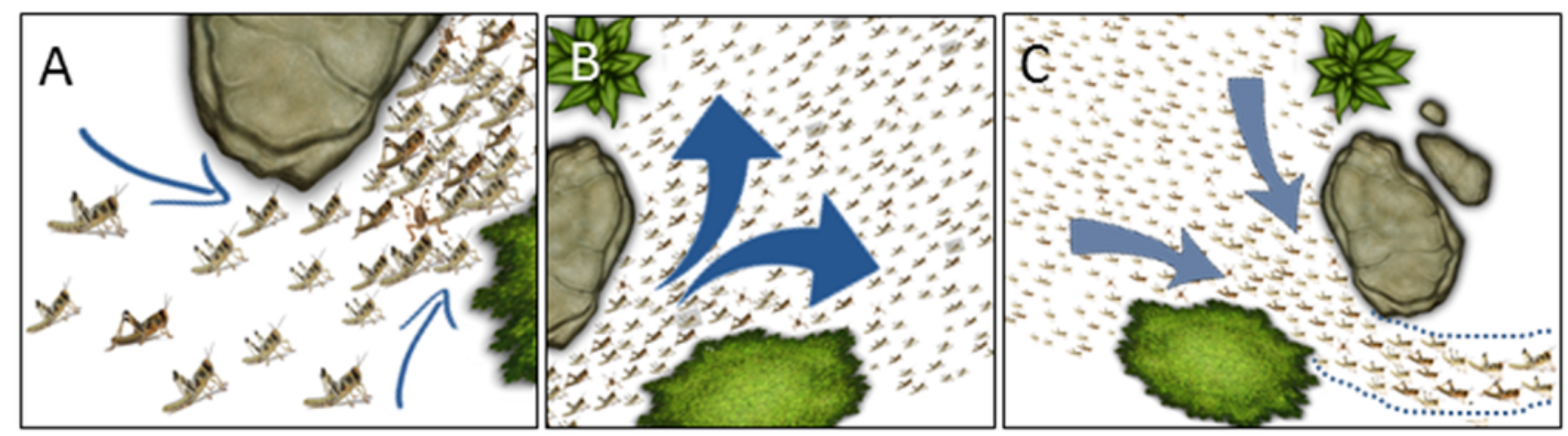

